\title{
Age Estimation Based on Skin Surface Images
}

\author{
Xiang-qian $\mathrm{HE}^{1}$, Ling-yu $\mathrm{LI}^{1}$, Zheng JIANG ${ }^{1}$ and $\mathrm{Hao} \mathrm{LI}^{2, *}$ \\ ${ }^{1}$ College of medical informatics, Chong-qing Medical University, China \\ ${ }^{2}$ Children's Hospital, Chong-qing Medical University, China \\ ${ }^{*}$ Corresponding author
}

Keywords: Age estimation, Image analysis, Feature extraction, Skin surface changes.

\begin{abstract}
This paper presents a model of detection age which quantifies the appearance changes of human skin at different stages of aging without traditional skeleton. We provide a novel method to extract age-related primitive features from skin surface images obtained by a digital camera, including skin surface heterogeneity parameters of the standard deviation of gray (Std) and the information entropy (Entropy) of skin images, the skin surface texture variations parameters of the mean area of the grids (MA) and the number of intersections (NI). Skin surface images of 97 individuals have been analyzed by our image processing program. The results show that the four parameters were statistically correlated with age, indicating their potential for the routine evaluation of skin aging.
\end{abstract}

\section{Introduction}

With aging, skin surface change its appearance gradually [1] due to intrinsic chronological factors and extrinsic environmental factors [2]. The most used techniques for measuring skin surface topography was the skin surface replicas analysis [3]. Although the replica analysis had advantages, they had many disadvantages which limit these methods for skin assessment. For example, this method was an indirect measure of the skin surface topography [4], which preparation of skin replicas was complicated and time-consuming, and the devices (e.g., laser profilometry) are usually expensive [5]. Therefore, a direct and flexible method without replicas for measuring skin surface topography was critically needed in skin aging research.

Image analysis method, based on images collected by portable digital devices, was a direct and convenient analysis method of skin surface topography. In this paper the image analysis method was proposed to extract features of the skin surface images to explore the information of aging.

\section{Materials and Methods}

\section{Skin Image Acquisition}

The test site was the middle part of the ventral of forearm. This site was chosen because it did not receive long-term sun exposure (less than the face [6], [7]) and was less stressed than the buttocks. Furthermore, the ventral forearm as the most commonly used skin site in dermatological research, was convenient to acquire skin surface images [8].

The forearm skin images were acquired by a flexible handheld device, which was a self-manufactured semi-finished product as shown in Fig. 1. The portable USB digital microscope (MDA2000) was produced by Future Optics Sci. \& Tech. Company 
(Hangzhou, Zhejiang Province, China). The circular polarizer (CPL, 37mm in diameter) was fixed to the transparent base of the former MDA2000 to weaken or eliminate the specular reflections from the skin surface effectively. All volunteers are consented to have their forearm photographed. When taking photos, their forearms were in a fixed position to relax the skin and muscles [9]. More than twenty images $(800 \times 600$ pixels) per subject were acquired with the acquisition device, at $40 \times$ magnification.

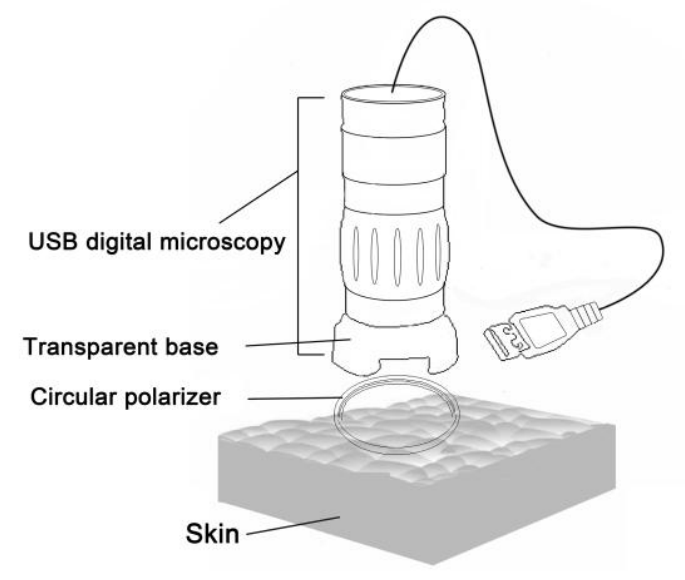

Fig. 1. Skin image acquisition device

\section{Skin Surface Feature Extraction}

Skin surface image features including color, shape, texture, and even the spatial relationships amid objects, had often been used in image retrieval [10], [11]. Here, we extracted the features (spatial relationships amid gray values and texture) of the skin surface images to explore the information of aging.

\section{Spatial Relationships Amid Gray Values}

Skin surface became rough as aging. Given that the skin image was an impression of the skin surface topography and the distribution of gray values of one image was proportional to the heterogeneity of the skin surface topography, the spatial relationships between the gray values were used to describe the heterogeneity of the skin surface topography. In this paper, the standard deviation of the gray values (Std) and the information entropy (Entropy) of grayscale skin image were employed to quantify the spatial relationships between the gray values.

\section{Texture Features}

The microscopic appearance of the skin surface was a net-like structure which was composed of primary and secondary lines [12]. These lines intersected each other to form polygons (grids) and intersections. With aging, the primary lines deepened and broadened while the number of secondary lines decreased [13]. Therefore, the mean area of grids (MA) and the number of intersections (NI) were two candidates to be calculated to quantify these skin texture variations since these variations were the most obvious indicators of the aging process.

\section{Algorithm}

An algorithm of automatic calculation of these four parameters (Std, Entropy, MA, and NI) was designed as following.

Step1: Image normalization and parameters (Std and Entropy) extraction 
The original skin image, of which the size is $800 \times 600$ pixels, was a truecolour RGB image. As the edge of the original skin image blurred, caused by the acquisition device, the original skin image was cropped to $400 \times 300$ pixels and the central area was remained. Then the cropped skin image was converted to a grayscale intensity image, which would be applied to calculate the parameter Std and Entropy.

The mean gray value of the skin image was expressed by:

$$
\text { Mean }=\frac{1}{M \cdot N} \sum_{j=0}^{N-1} \sum_{i=0}^{M-1} \operatorname{Gray}(i, j)
$$

where $\operatorname{Gray}(i, j)$ was the gray value of pixel $(i, j)$, and $M, N$ represent height and width of the image, respectively. Then parameter Std was defined in the following way:

$$
S t d=\sqrt{\frac{1}{M \cdot N} \sum_{j=0}^{N-1} \sum_{i=0}^{M-1}(\operatorname{Gray}(i, j)-\text { Mean })^{2}}
$$

The parameter Entropy was calculated by:

$$
\text { Entropy }=-\sum_{1=0}^{\mathrm{L}-1} p(I) \cdot \log _{2} p(I)
$$

where $p(1)$ represented the probability distribution of grayscale 1 , and $\mathrm{L}$ ( $\mathrm{L} \geq 1$ ) represented the number of total grayscales.

Step2. Image enhancement

The Contrast-Limited Adaptive Histogram Equalization (CLAHE) was applied to the grayscale image to enhance the contrast and to prevent noise over-amplification[14]. The median filtering process was utilized for reducing image noise and non-uniform background due to the color stemmed from minute blood vessels or the brightness from the oily skin surface. Moreover, the morphological reconstruction, including opening-by-reconstruction and closing-by-reconstruction, were used to further improve the image.

Step 3: Image binarization and thinning

To detect the skin texture, Otsu's method was used to get a binary image. The binary image was thinned and a single pixel-wide image which will facilitate the computation of the texture parameter was achieved.

Step 4: Texture parameter calculation

The number of grids was counted before calculating parameter MA. A large number of burrs in the thinned image were removed to compute the number of grids (Fig. 2). Then parameter MA was computed based on (4).

$$
M A=\frac{M \cdot N}{n}
$$

where $\mathrm{n}$ was the number of grids in the skin image. $\mathrm{M}, \mathrm{N}$ represented the height and width of the image, respectively.

Intersection was defined as a single point intersected by three or more texture lines. When the thinned image was used to detect the intersections, the burrs around the edge of the image may cause pseudo intersections, so we partly pruned the burrs to detect the intersections accurately (Fig. 3(a)). The detecting result of intersections was shown in Fig. 3(b). To further improve precision, a group of detected pixels in a defined area was 
regarded as one intersection. Fig. 3(c) showed the combined result of intersections with the CLAHE image.

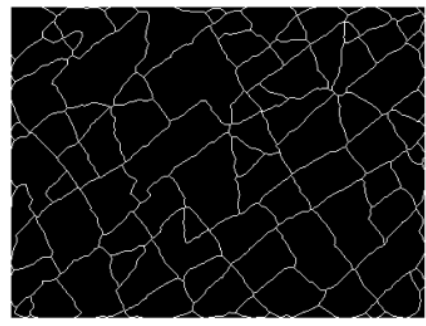

Fig. 2. The thinned grids image

(a)

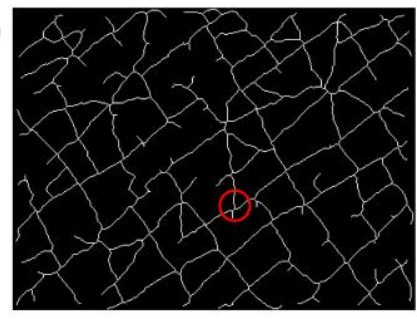

(b)

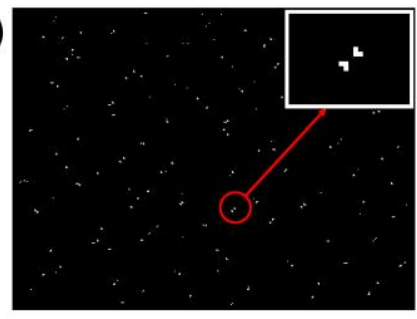

(c)

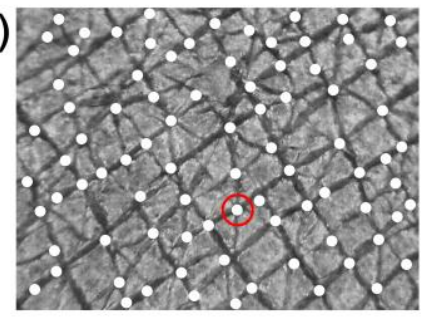

Fig. 3. The processing of intersections detection. (a) Removing burrs. (b)The result of intersections detection. (c) The detection result of intersections combines with the CLAHE image.

\section{Experimental Results}

\section{Human Subjects}

A total of 58 female and 39 male healthy Chinese volunteers aged 20-70s were recruited for this experiment. They all live in southwest China and are engaged in indoor work. Besides, their body mass indexes (BMI) are within a certain limit $(18.0<\mathrm{BMI}<25.0)$ and subjects who were addicted to smoking or drinking were excluded.

\section{Image Processing Results}

Fig. 4 demonstrated the processing results of skin surface images of the ventral forearm of two male volunteers with different ages. It showed that skin texture lines widened and decreased with age. Concurrently, the number of grids and intersections decreased. The histogram of grayscale skin image of 60.6 years old distributed more discretely than that of 40.1 years old, which means the elderly subject have rougher skin surface than the young one.

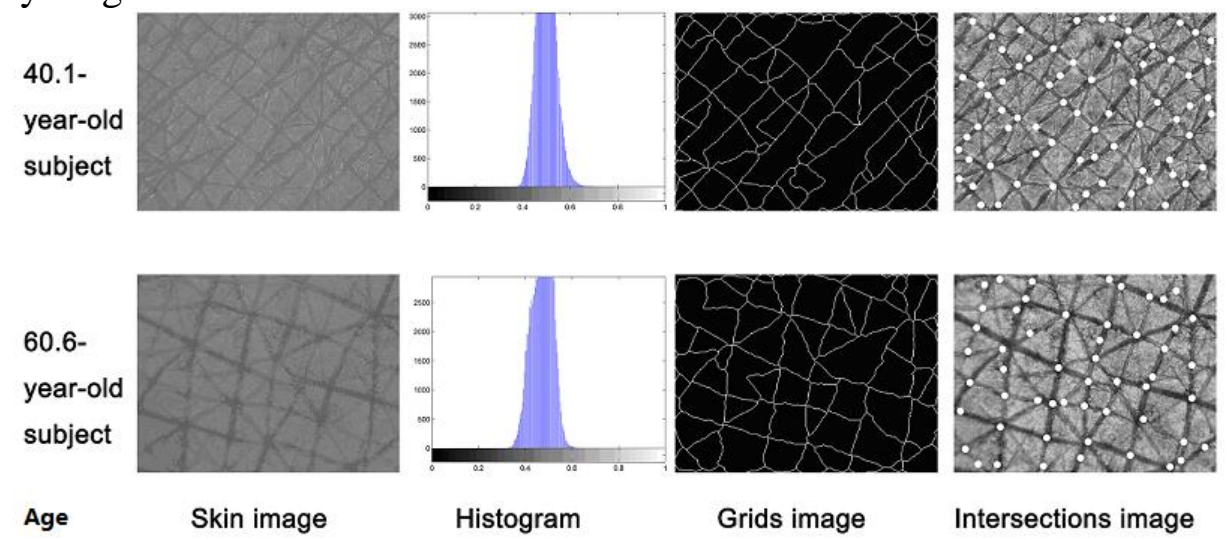

Fig. 4. Image features extraction results of two volunteers. 


\section{Statistical Analysis}

The correlation between age and each parameter was evaluated by $\mathrm{R}(\mathrm{R}$ is available at https://www.r-project.org/).

The values of the investigated parameters are plotted versus subjects' age and the regression lines are shown in Fig. 5. The standard deviation of the gray values (Std), the information entropy (Entropy), and the mean area of grids (MA) all increased with age, while the number of intersections (NI) decreased significantly with age. In detail, the correlation coefficient between age and each parameter of female was higher than that of male. And NI showed the very high correlation with age when compared with other three parameters $(\mathrm{r}=-0.865, \mathrm{P}<0.01$ in female; $\mathrm{r}=-0.785, \mathrm{P}<0.01$ in male) (Fig. 6).
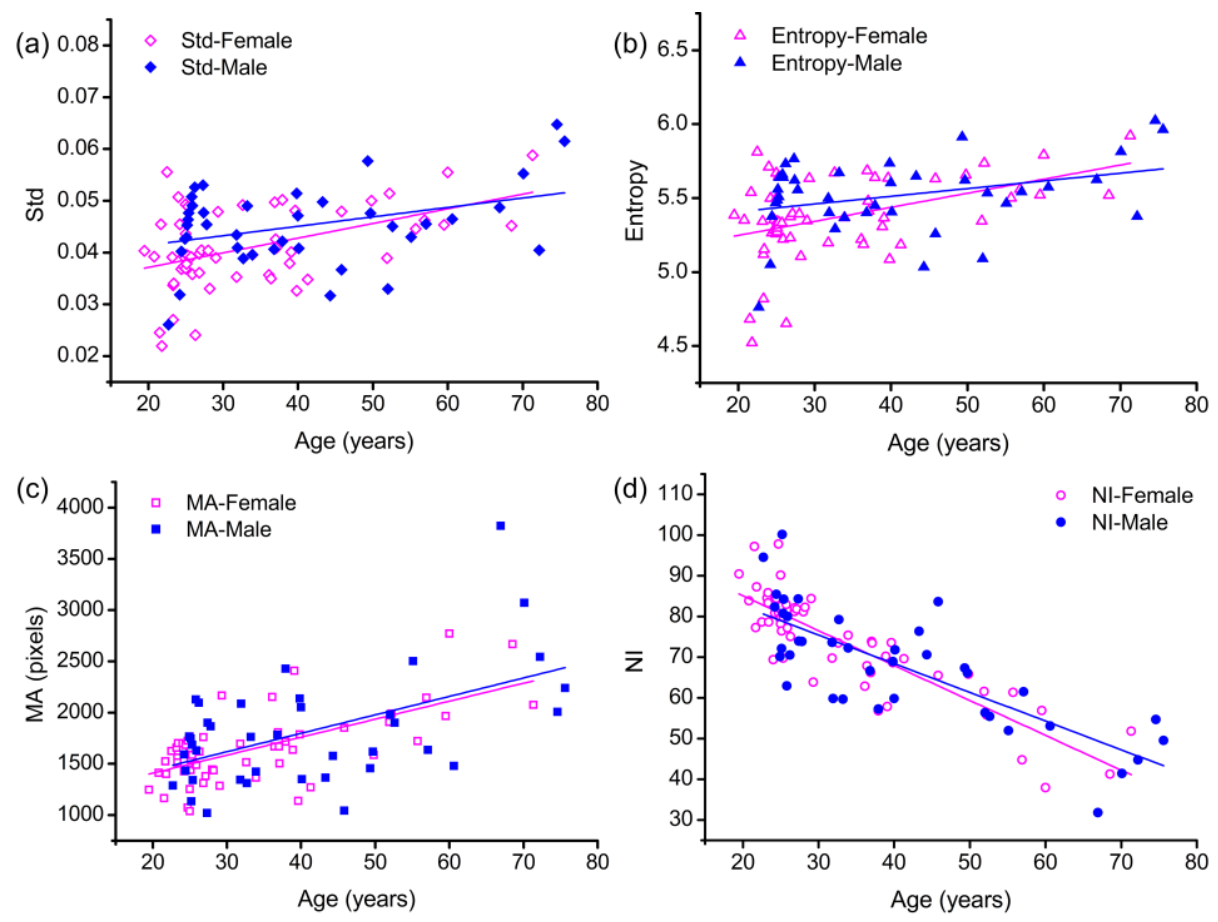

Fig. 5. linear regression (a) Std (b) Entropy, for (c) MA and (d) NI with age

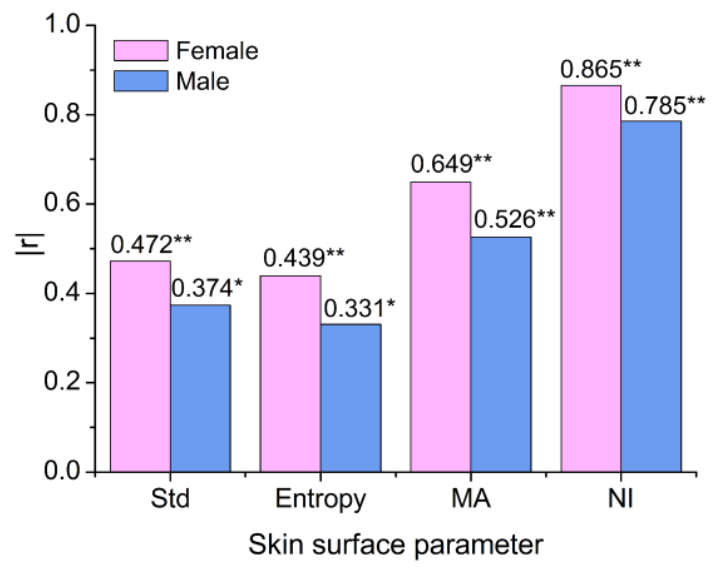

Fig. 6. The correlation coefficient between parameters and age.

$* \mathrm{P}<0.05 ; * * \mathrm{P}<0.01 ; \mathrm{r}$, correlation coefficient. 


\section{Discussion}

With age increasing, the skin surface topography changes its appearance. For example, the primary lines deepen and broaden while the secondary lines distort or even disappear [5]. To establish an objective and quantitative assessment of these skin surface variations, skin surface parameters (Std, Entropy, MA, and NI) related to skin image primitive features are mainly investigated in this paper.

Results show that all the investigated parameters are correlated with age (Fig. 5, 6), indicating that these parameters would be applied to assess the variations of aging skin. MA and NI were strongly correlated with age in comparison with Std and Entropy (Fig. 6), probably because the calculation of these two parameters was based on gray values which were sensitive to the light intensity and direction. And the correlation coefficient of each parameter with age of female was higher than that of male, which may result from the inherent difference that men have a thicker dermis than women [6]. Additionally, another factor that may affect the correlation difference between male and female is that men tend to have more physical work than women, which may accelerate men's skin aging.

Furthermore, NI showed an extremely high correlation with age when compared with other three parameters (Fig. 6), thus it deserved special attention. Ryu et al. [12] have shown through the use of skin replicas that the number of 'star configurations' (intersections) on left cheek skin decreases with age, a result that agrees with our data. To verify the accuracy of the automatic detection and calculation of intersections in our study, we also counted the intersections manually. The significant correlation between the number of intersections counted automatically and that counted manually $(r=0.986$, $\mathrm{P}<0.001$ ), as shown in Fig. 7, indicated that the detection and calculation results of our program were in line with the results human perceived in RGB skin image. In addition, the difference between them was not statistically significant based on the result of Paired T-test $(\mathrm{P}=0.119)$, which indicated our processing program can be applied to count the number of intersections automatically. All these findings suggested that NI would be a potential parameter for characterizing skin aging.

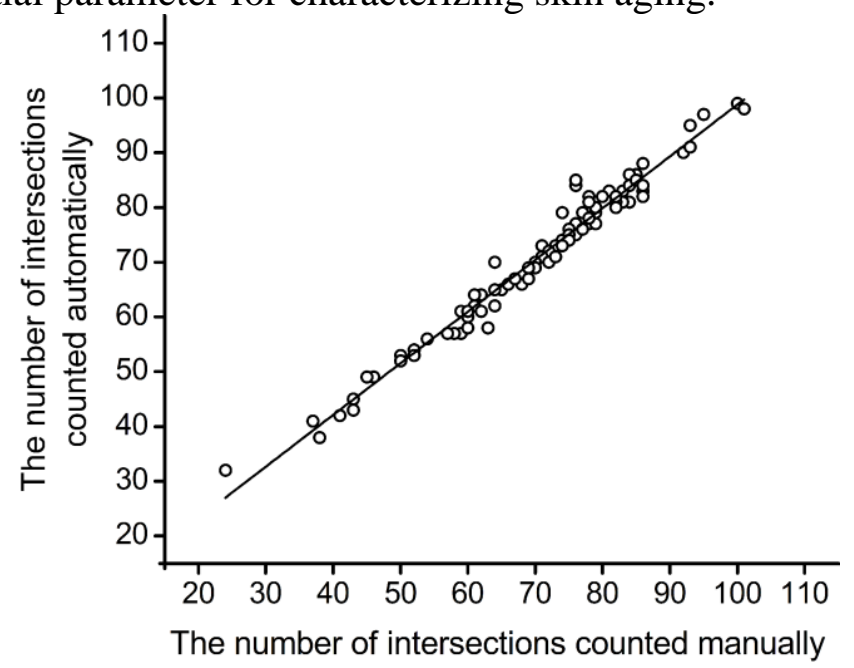

Fig. 7. Correlation between the number of intersections counted automatically and that counted manually.

Some studies [6], [15] reported the effect of a high body mass index (BMI) was associated with looking younger than one's age. In this research, we noticed that an individual with higher BMI had a denser skin texture appearance, e.g., smaller grid areas and more intersections, so we supposed that the skin texture parameters were 
proportional to BMI. Thus, we multiplied the parameter MA by BMI (MA*BMI) and divided the parameter NI by BMI (NI/BMI) to eliminate effects of BMI, and then explored the relationships between these two parameters (MA*BMI and NI/BMI) with age. Consequently, the statistical results revealed that $\mathrm{MA} * \mathrm{BMI}$ and NI/BMI were significantly correlated with age (Table I). Hence, a study with larger sample of subjects who have the same age but different BMI will be considered in the future work to test and verify the proportional relation between BMI and the four investigated skin surface parameters.

Other factors, such as smoking, drinking, are also reported to have significant effects on skin aging [16]. In this paper, all the subjects had no smoking and drinking history. Environmental factors, such as cumulative ultraviolet radiation [17], are known to cause photoaging on the skin. Many studies reported a distinction between chronoaging (which referred to the physiological chronological changes) and photoaging. Therefore, a sun exposed site (e.g., the dorsal forearm), and a sun protected site (e.g., the upper inner arm) will be evaluated with our skin surface parameters in the future.

In summary, we have developed an image analysis method based on extracting features of the skin surface image to assess skin surface topography variations due to skin aging, which was direct and practical compared with the replicas methods. The investigation of the parameters (Std, Entropy, MA, and NI) showed their potential for the evaluation of skin aging. Coupling with common skin parameters, such as skin hydration, skin gloss, skin elasticity, the proposed method might be a reasonable tool in cosmetology, dermatological research and practice.

Table 1. Correlation of parameters affected by BMI with age

\begin{tabular}{llll}
\hline \hline Gender & Parameter & $\mathrm{r}$ & P-value \\
\hline Female & MA*BMI & $0.716^{* * *}$ & 0.000 \\
& NI/BMI & $-0.852^{* *}$ & 0.000 \\
Male & MA*BMI & $0.514^{* * *}$ & 0.001 \\
& NI/BMI & $-0.723^{* *}$ & 0.000 \\
\hline \hline
\end{tabular}

**. Correlation is significant at the 0.01 level (2-tailed).

\section{Acknowledgement}

This research was financially supported by the Chongqing Science and Technology Commission, Chongqing People's Municipal Government (number cstc2013yykfA0095), and Chongqing Medical University (number 2014A004).

\section{References}

[1] E. M. Wurm, C. Longo, C. Curchin, H. P. Soyer, T. W. Prow, and G. Pellacani, "In vivo assessment of chronological ageing and photoageing in forearm skin using reflectance confocal microscopy," The British journal of dermatology, vol. 167, pp. 270-279, Aug 2012. 
[2] G. J. Fisher, S. Kang, J. Varani, Z. Bata-Csorgo, Y. Wan, S. Datta, et al., "Mechanisms of photoaging and chronological skin aging," Arch. Dermatol., vol. 138, pp. 1462-1470, 2002.

[3] J. Lagarde, C. Rouvrais, and D. Black, "Topography and anisotropy of the skin surface with ageing," Skin Res. Technol., vol. 11, pp. 110-119, 2005.

[4] P. R. Bargo and N. Kollias, "Measurement of skin texture through polarization imaging," The British journal of dermatology, vol. 162, pp. 724-731, Apr 2010.

[5] Y. Zou, E. Song, and R. Jin, "Age-dependent changes in skin surface assessed by a novel two-dimensional image analysis," Skin research and technology : official journal of International Society for Bioengineering and the Skin, vol. 15, pp. 399-406, Nov 2009.

[6] M. B. Purba, A. Kouris-Blazos, N. Wattanapenpaiboon, W. Lukito, E. Rothenberg, B. Steen, et al., "Can skin wrinkling in a site that has received limited sun exposure be used as a marker of health status and biological age?," Age Ageing, vol. 30, pp. 227-234, 2001.

[7] S. G. Park, Y. D. Kim, J. J. Kim, and S. H. Kang, "Two possible classifications of facial skin type by two parameters in Korean women: sebum excretion rate (SER) and skin surface relief (SSR)," Skin Res. Technol., vol. 5, pp. 189-194, 1999.

[8] C. Trojahn, G. Dobos, M. Schario, L. Ludriksone, U. Blume-Peytavi, and J. Kottner, "Relation between skin micro-topography, roughness, and skin age," Skin research and technology : official journal of International Society for Bioengineering and the Skin, vol. 21, pp. 69-75, Feb 2015.

[9] F. Kautzky, M. W. Dahm, M. Drosner, L. D. Köhler, H. J. Vogt, and S. Borelli, "Direct profilometry of the skin: its reproducibility and variability," J Eur Acad Dermatol Venereol, vol. 5, pp. 15-23, 1995.

[10] Content Based Image Retrieval Using Color, Shape and Texture

[11] A New Method for Content based Image Retrieval using Primitive Features

[12] J. H. Ryu, Y. K. Seo, Y. C. Boo, M. Y. Chang, T. J. Kwak, and J. S. Koh, "A quantitative evaluation method of skin texture affected by skin ageing using replica images of the cheek," International journal of cosmetic science, vol. 36, pp. 247-252, Jun 2014.

[13]K. De Paepe, J.-M. Lagarde, Y. Gall, D. Roseeuw, and V. Rogiers, "Microrelief of the skin using a light transmission method," Arch. Dermatol. Res., vol. 292, pp. 500-510, 2000.

[14] S. M. Pizer, E. P. Amburn, J. D. Austin, R. Cromartie, A. Geselowitz, T. Greer, et al., "Adaptive histogram equalization and its variations," Comput Vis Graph Image Process, vol. 39, pp. 355-368, 1987.

[15]C. Guinot, D. J.-M. Malvy, L. Ambroisine, J. Latreille, E. Mauger, M. Tenenhaus, et al., "Relative contribution of intrinsic vs extrinsic factors to skin aging as determined by a validated skin age score," Arch. Dermatol., vol. 138, pp. 1454-1460, 2002.

[16]A. Morita, "Tobacco smoke causes premature skin aging," Journal of dermatological science, vol. 48, pp. 169-175, Dec 2007. 
[17] M. Farage, K. Miller, P. Elsner, and H. Maibach, "Intrinsic and extrinsic factors in skin ageing: a review," Int. J. Cosmetic Sci., vol. 30, pp. 87-95, 2008. 